\title{
L'impact de la création des agences d'exécution sur le Civil Service : décentralisation, marchandisation et conséquences constitutionnelles
}

The Impact on the Civil Service of the Creation of Executive Agencies:

Decentralisation, Marketisation and Constitutional Consequences

\section{Nicholas Sowels}

\section{(2) OpenEdition}

Journals

\section{Édition électronique}

URL : http://journals.openedition.org/rfcb/3412

DOI : $10.4000 /$ rfcb.3412

ISSN : 2429-4373

Éditeur

CRECIB - Centre de recherche et d'études en civilisation britannique

\section{Édition imprimée}

Date de publication : 1 avril 2005

ISBN : 2-911580-20-6

ISSN : 0248-9015

\section{Référence électronique}

Nicholas Sowels, «L'impact de la création des agences d'exécution sur le Civil Service:

décentralisation, marchandisation et conséquences constitutionnelles », Revue Française de Civilisation Britannique [En ligne], XIII-2 | 2005, mis en ligne le 01 avril 2005, consulté le 10 avril 2020. URL : http:// journals.openedition.org/rfcb/3412 ; DOI : https://doi.org/10.4000/rfcb.3412

Ce document a été généré automatiquement le 10 avril 2020.

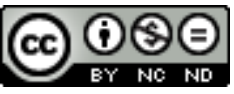

Revue française de civilisation britannique est mis à disposition selon les termes de la licence Creative Commons Attribution - Pas d'Utilisation Commerciale - Pas de Modification 4.0 International. 


\title{
L'impact de la création des agences d'exécution sur le Civil Service : décentralisation, marchandisation et conséquences constitutionnelles
}

\author{
The Impact on the Civil Service of the Creation of Executive Agencies: \\ Decentralisation, Marketisation and Constitutional Consequences
}

Nicholas Sowels

\section{NOTE DE L'AUTEUR}

Je tiens à remercier Roxana NADIM pour ses commentaires sur ce texte. La responsabilité de toute erreur reste évidemment la mienne.

1 Comme tous les domaines du secteur public britannique, le Civil service (l'administration centrale) a été profondément réformé depuis l'arrivée au pouvoir des conservateurs en 1979. Il s'agit d'une véritable restructuration des services centraux de l'État, qui s'inscrit dans une politique générale pour restreindre et modifier le rôle de l'État et des services publics. Le premier volet des réformes conservatrices de l'administration est caractérisé par la recherche d'économies. Il s'agit notamment de réduire le nombre d'agents par le non-renouvellement de postes et d'introduire au Civil Service des pratiques de gestion inspirées de l'entreprise privée. Ce n'est que le troisième gouvernement Thatcher (1987-1990) qui lance la véritable restructuration du Civil Service, par la création des agences d'exécution (executive agencies). Les deux gouvernements Major (1990-1992 et 1992-1997) approfondissent ces réformes. Ils développent les relations contractuelles qui existent dorénavant entre le pouvoir exécutif central et les agences ainsi qu'entre les services publics et les citoyens. Ils consolident les nouvelles méthodes de gestion et mettent en concurrence des services internes de l'administration avec des sous-traitants privés. L'orientation générale de 
ces réformes est reprise par les deux gouvernements Blair (1997-2001 et 2001- ), bien qu'ils soient moins critiques de l'action publique que leurs prédécesseurs. Cet article analyse les traits fondamentaux de ces réformes ainsi que leur impact sur la position constitutionnelle du Civil Service et ses relations avec les citoyens.

\section{Les réformes Thatcher de l'administration centrale}

2 Les réformes Thatcher du Civil Service sont entreprises pour plusieurs raisons. Tout d'abord, les gouvernements Thatcher cherchent à maitriser les dépenses publiques. Réduire le nombre de Civil Servants devient le levier principal par lequel le gouvernement cherche à faire des économies. Cette politique s'inscrit dans une stratégie plus globale qui consiste à diminuer l'action publique dans l'économie et à renforcer le rôle du marché dans l'organisation des services publics. Une telle stratégie commence à être formulée pendant les années 1970. Elle se fonde sur une réflexion profonde engagée par une "Nouvelle droite " qui se constitue en Grande-Bretagne, autour de Mme Thatcher et ses alliés. Cette Nouvelle droite met en cause tous les aspects du « consensus d'après-guerre », basé sur la politique économique keynésienne, l'État-providence et l'intervention publique dans l'économie. Se référant explicitement aux diverses théories néo-classiques économiques qui critiquent l'action des pouvoirs publics, la Nouvelle droite élabore progressivement un programme détaillé pour réformer le rôle de l'État et des services publics.

\section{Les premières réformes des gouvernements Thatcher}

Dans un premier temps, les mesures prises pour réformer l'administration centrale sont restreintes. Il s'agit surtout de réduire le nombre d'employés et de mettre en place des enquêtes (Raynor Scrutinies) sur l'efficacité de certains services. Ainsi, en mai 1980, Margaret Thatcher annonce à la Chambre des communes un plan visant à réduire à 630000 le nombre de fonctionnaires, soit une compression de 100000 par rapport à l'effectif dont elle a hérité en mai 1979 (voir Tableau 1)1. Cette diminution est réalisée par le gel des recrutements, alors que les départs volontaires (à la retraite et autres) se chiffrent à environ 80000 par an. C'est une politique qui va se poursuivre tout au long des années 1980 et 1990 et qui voit le nombre de civil servants baisser jusqu'à 459600 en 1997.

Tableau 1 : Les effectifs du Civil Service et les dépenses publiques globales pour l'administration centrale (en \% du PIB)

\begin{tabular}{|l|l|l|l|l|}
\hline & 1979 & 1988 & 1997 & 2002 \\
\hline Emplois* & 732300 & 597814 & 459600 & 490420 \\
\hline $\begin{array}{l}\text { Dont agences d'exécution } \\
(\% \text { du total) }\end{array}$ & & & $\begin{array}{l}285530 \\
62 \%\end{array}$ & $\begin{array}{l}262810 \\
53 \%\end{array}$ \\
\hline $\begin{array}{l}\text { Total organismes Next Steps } \\
(\% \text { du total) }\end{array}$ & & 350410 & 355660 \\
$76 \%$ & $73 \%$ \\
\hline
\end{tabular}




\begin{tabular}{|l|l|l|l|l|}
\hline Nombre d'agences & & & 124 & 127 \\
\hline & & & & \\
\hline Dépenses en \% du PIB (exercice) & & $1988 / 9$ & $1997 / 8$ & $2002 / 3$ \\
\hline Administration centrale et dépenses associées** & 1,2 & 1,0 & 1,3 \\
\hline
\end{tabular}

*Emplois équivalents de temps plein.

** Ce chiffre comporte les transferts budgétaires nets à l'Union européenne.

Sources: Civil Service Statistics (série annuelle), HM Treasury, Public Expenditure Statistical Analyses (série annuelle), <http://www.cabinetoffice.gov.uk/>, 5/11/2004.

4 L'impact de ces réformes et le langage de la gestion, qui est appliqué de plus en plus au Civil Service, restent néanmoins limités. Il est estimé, par exemple, qu'en six ans les Rayner Scrutinies ${ }^{2}$, qui enquêtent sur le fonctionnement des activités spécifiques du Civil Service, ont entraîné des économies de seulement 600 millions de livres par an ${ }^{3}$. De même, les dépenses évitées par la suppression des postes sont en partie perdues car l'administration centrale se trouve obligée d'acheter un certain nombre de services qu'elle ne peut plus effectuer en interne. En somme, la réforme du Civil Service jusqu'au milieu des années 1980 est restreinte.

\section{La création des agences d'exécution}

C'est alors, au milieu des années 1980, que Margaret Thatcher se tourne vers Robin Ibbs, successeur de Derek Rayner à l'Efficiency Unit, pour effectuer une étude du Civil Service et élaborer de nouvelles propositions de réforme. Le Rapport Ibbs, Improving Management in Government : the Next Steps (Améliorer la gestion de l'État: les prochaines étapes), réitère l'urgence de la mise en œuvre des mesures pour améliorer le rapport qualité-prix des services ${ }^{4}$. Mais surtout, le rapport constate que les fonctions de l'administration centrale sont très diverses, ce qui rend l'organisation historique de l'administration, basée sur l'uniformité des structures, peu appropriée ${ }^{5}$. Par conséquent, le Rapport Ibbs propose une série de mesures qui visent à démembrer les ministères pour créer des services autonomes, notamment :

-L'éclatement des ministères en "services centraux", responsables de la formulation de la politique gouvernementale et en " agences d'exécution » (executive agencies) responsables de la mise en œuvre de la politique gouvernementale et de tâches administratives ;

- la nomination de directeurs-généraux (chief executives) à la tête des agences, ayant la responsabilité opérationnelle de leur agence ;

- le contrôle de la performance des agences par rapport à des objectifs élaborés et spécifiés par les ministres ;

- l'élaboration d'un document cadre (framework document) qui stipule clairement les objectifs et les moyens accordés à l'agence.

L'élément fort de ces propositions se trouve dans l'autonomie des agences. Une fois le document cadre élaboré et signé par le ministre et le directeur d'agence, ce dernier a l'autonomie opérationnelle pour exécuter ses fonctions. Il peut gérer son budget d'une manière indépendante, selon les besoins spécifiques de son agence, embaucher $d u$ personnel si nécessaire et établir de nouveaux contrats de travail de droit privé. Une 
telle autonomie permet de développer au sein de l'administration les comportements propres au secteur privé.

7 La création des agences avance rapidement et elle est globalement achevée en 1997, quand 364136 civil servants sur 475 340, soit 77\% des employés de l'administration centrale, travaillent dans des agences d'exécution ou organismes similaires. (au $1^{\mathrm{er}}$ avril 2002, il existait 127 agences, dont 92 sous la tutelle de l'administration centrale à Londres (Whitehall), les autres agences étant sous la tutelle des autorités exécutives d'Écosse, du Pays de Galles et d'Irlande du Nord) ${ }^{6}$. En dépit de leur similitude statutaire, la taille, le fonctionnement et les missions des agences sont très variés. La plus grande en 2002, le Jobcentre Plus, est responsable du versement des prestations sociales (allocations chômages etc.) et du reclassement des chômeurs. Elle compte 90000 employés. À l'opposé, le Wilton Park Conference Centre n'a qu'un effectif de 50 personnes.

\section{La poursuite des réformes par les gouvernements Major et Blair}

8 Les gouvernements Major achèvent la politique de création des agences. Puis ils développent la contractualisation des relations entre les ministères et les agences, d'une part et le secteur public (y compris le Civil Service) et les citoyens, d'autre part. En outre, les gouvernements Major cherchent à susciter la concurrence à l'intérieur de l'administration centrale. Quant aux gouvernements Blair, ils reprennent les grandes lignes des réformes conservatrices tout en s'appuyant sur un discours politique plus favorable au secteur public que celui des conservateurs. Ils cherchent également à pallier les carences fonctionnelles des services publics qui découlent de leur éclatement structurel. Toutefois, dans leur quête pour moderniser l'action publique, les travaillistes poursuivent les réformes de leurs prédécesseurs.

\section{Les indicateurs de performance et la contractualisation dans le Civil Service}

9 Les gouvernements Major mènent à terme la création des agences d'exécution, qui est accompagnée par une contractualisation importante des relations entre les agences d'exécution et leurs ministères de tutelle. Parallèlement, les conservateurs approfondissent la politique d'identification et donc la meilleure maîtrise des coûts de l'administration centrale, par le biais de l'élaboration d'indicateurs de performance. De tels indicateurs sont progressivement mis en place durant la seconde moitié des années 1980. Ils sont utilisés pour mesurer et ainsi mieux contrôler toutes les activités du secteur public et pour établir des objectifs détaillés que les organismes publics doivent atteindre. Selon S. Lewis et J. Jones, il est possible d'identifier des indicateurs qui quantifient le traitement des dossiers/objets, le traitement des cas individuels, le travail en projet, les fonctions d'urgence, la maintenance routinière et le traitement personnalisé des dossiers individuels ${ }^{7}$.

10 Pris dans son ensemble, le recours à des documents cadres et aux indicateurs de performance mène à une contractualisation des relations entre le pouvoir exécutif central (core executive) et les agences d'exécution. Certes, il ne s'agit pas de véritables contrats établis entre parties privées, soumis au droit commercial. Néanmoins, la façon 
dont les responsabilités des uns et des autres sont explicitées crée des possibilités de recours et de sanctions à la disposition des ministères de tutelle en cas de non-respect des engagements de la part des agences.

11 Cette contractualisation de l'administration centrale est aussi renforcée à partir de 1991 avec l'élaboration de la Charte du citoyen (Citizen's Charter) qui est établie pour l'ensemble du secteur public, y compris l'administration centrale. Les objectifs généraux de ce dispositif sont d'améliorer la qualité des services et d'élargir les choix des citoyens. Plus spécifiquement, la Charte repose sur des principes-clefs, tels que: l'élaboration de critères de qualité ; le choix et la consultation; la qualité de l'accueil ; la correction des erreurs ; l'amélioration du rapport qualité-prix (value for money) dans la réalisation des services ${ }^{8}$. La Charte nationale est ensuite déclinée et adaptée aux différents services publics. Il existe alors quarante Chartes principales qui s'appliquent aux grands domaines du secteur public et plus de dix mille Chartes spécifiques par établissement ou unité individuelle9. Le premier gouvernement Blair reprend l'essentiel de ce programme, même s'il le refaçonne et le renomme Service First. Plus tard, la structure nationale de la Charte sera effacée par d'autres mesures pour sonder la satisfaction des utilisateurs afin de rendre les services publics plus sensibles aux attentes des citoyens. Toutefois, un système pour évaluer, noter et ainsi récompenser les organismes publics individuels est maintenu (Charter Mark).

\section{La mise en concurrence des services internes avec les prestataires privés}

Le deuxième axe des réformes du Civil Service par les gouvernements Major concerne la mise en concurrence des services internes de l'administration avec des prestataires privés. Dans son Livre blanc de 1991, Competing for Quality, le gouvernement constate que «les entreprises du secteur privé se sont concentrées de plus en plus sur leurs activités de bases...et qu'elles achètent les services des sous-traitants qui leurs fournissent des idées nouvelles, une plus grande flexibilité et un niveau d'expertise plus élevé par rapport aux services internes $»^{10}$. Le Livre blanc note aussi que les organismes publics commencent à faire la même chose et affirme que le gouvernement cherche à renforcer ce processus. En particulier, le Livre blanc annonce que le gouvernement souhaite développer la concurrence en ce qui concerne les services professionnels de l'administration centrale (services de conseils, d'audit, d'inspection, etc.) ${ }^{11}$. Dorénavant, l'administration doit lancer des appels d'offre pour «tester » le rapport qualité-prix de ses propres services par rapport aux prestataires externes (market testing).

13 Pour le gouvernement, cette mise en concurrence des services (des ministères ou des agences) avec le secteur privé offre trois avantages ${ }^{12}$. Pemièrement, elle peut réduire les coûts de fonctionnement d'une activité, car les entreprises privées ont la possbilité de bénéficier d'économies d'échelles ou d'autres avantages dûs à une meilleure spécialisation de production. Deuxièmement, la mise en concurrence des services, ainsi que la formulation d'appels d'offres nécessitent une plus grande précision des tâches administratives à accomplir. Ceci permet une meilleure identification des coûts de fonctionnement, ce qui facilite leur réduction. Troisièmement, la mise en concurrence des services renforce le processus de contractualisation qui encadre de plus en plus les activités du Civil Service. 


\section{La « modernisation » de l'administration centrale par les gouvernements Blair} mouvance que celles de ses prédécesseurs, et ils poursuivent des réformes cherchant à accroître la productivité de l'administration centrale, bien que le discours travailliste envers les fonctionnaires devienne moins hostile. Ainsi, le premier gouvernement Blair développe de nouvelles procédures budgétaires pour le secteur public. Celles-ci sont caractérisées par la mise en place d'une planification des budgets ministériels sur trois ans, la séparation plus claire des dépenses courantes et des dépenses d'investissement et les possibilités de reporter des fonds ministériels d'une année à l'autre ${ }^{13}$.

Cette nouvelle budgétisation est accompagnée par l'élaboration d'Accords de service public (PSAs - Public Service Agreements) qui stipulent les objectifs principaux de chaque ministère et des objectifs interministériels. Les PSAs précisent aussi des «objectifs de performance " (performance targets) spécifiques afin de mesurer la productivité et l'efficacité des services. Le Livre blanc du premier gouvernement Blair sur la «modernisation des services publics » (Modernising Government), publié en 1998, prône une approche globale vis-à-vis du fonctionnement des services publics, qui doivent être résolument orientés vers les citoyens ${ }^{14}$. Quant au Civil Service, Modernising Government reprend la politique de formulation des objectifs afin d'améliorer le contact entre l'administration et les citoyens. Les ministères et les agences doivent élaborer des objectifs pour :

- répondre rapidement aux courriers ;

- honorer les horaires de rendez-vous fixés avec le public;

- répondre rapidement et efficacement aux appels téléphoniques ;

- donner des informations claires et simples sur l'organisme en question ;

- établir des procédures de recours;

- faire tout leur possible pour assurer que tout le monde puisse accéder aux services ${ }^{15}$.

Hormis les efforts affichés pour mieux servir le public, le thème de « l'interconnexion des services publics » (joined-up government) est souligné par le Livre blanc. Il s'agit en effet d'une problématique fondamentale au bon fonctionnement des services publics, car les réformes des années 1980-1990 et la création des agences ont dispersé les responsabilités opérationnelles des organismes publics: la contractualisation des relations entre les agences et leurs ministères de tutelle limite, de fait, les responsabilité de chaque agence à sa propre mission, clairement définie dans son document cadre. Par conséquent, les relations verticales (entre les agences et leur ministère de tutelle) et horizontales (entres les agences et les autres services de l'administration centrale) ont tendance à diminuer, car chaque unité se concentre sur ces tâches spécifiques.

Cette dispersion des responsabilités a été effectuée pour rendre le fonctionnement de l'administration plus souple et concurrentiel, plus varié et donc aussi plus sensible aux besoins des consommateurs. Mais il en résulte des structures éclatées, dont il est plus difficile de contrôler et de coordonner le travail. Ainsi, en 2002, un audit majeur des agences entrepris par les services du Premier ministre critique le manque de coordination qui existe entre les ministères et les agences. L'audit préconise alors un certain nombre de mesures pour améliorer cette coordination, comme, par exemple, 
l'organisation de réunions plus régulières entre les ministères et les agences, une mise à jour annuelle du document cadre, etc. ${ }^{16}$.

La coordination du travail effectué par l'administration centrale est néanmoins devenue plus complexe car le gouvernement travailliste cherche aussi à renforcer le rôle des prestataires privés dans la réalisation des services de l'administration centrale. Ceci oblige les organismes publics (ministères de tutelle ou agences) à rédiger des contrats détaillés avec des sous-traitants privés et à vérifier le respect de tels contrats, ce qui est souvent difficile à faire, notamment, par exemple, quand il s'agit de veiller au respect des critères de performance concernant la qualité des services. De même, le gouvernement Blair approfondit le recrutement des employés issus du secteur privé. Il s'agit encore une fois d'apporter des compétences diversifiées au Civil Service, mais le recours aux cadres externes implique également le recrutement d'agents qui n'ont pas la même culture du service public que les Civil Servants : si ces agents sont plus efficaces dans les domaines techniques, il leur manque parfois une vision globale sur le fonctionnemment de l'administration et son rôle plus général dans la mise en œuvre de la politique publique.

Par la suite, dans un discours détaillé prononcé en février 2004 sur l'avenir de l'administration, Tony Blair confirme sans ambiguïté la poursuite des réformes lancées depuis les années 1980. Certes, il réitère l'importance des valeurs traditionnelles du Civil Service issues du Rapport Northcote-Trevelyan de 1854, telles que l'intégrité, l'impartialité et le mérite. De même, Tony Blair dit que 'I do not believe in a minimalist state. But I don't believe in Big Government either. I believe in Enabling Government'.

Toutefois, pour que l'administration centrale devienne plus entreprenante, il faut la rendre moins rigide. Il faut qu'elle acquière le goût de l'aventure comme il existe dans le secteur privé. Plus concrètement, Tony Blair annonce qu'il faut développer :

- un centre stratégique plus petit ;

- un Civil Service ayant des compétences professionnelles et de spécialité ;

- un Civil Service ouvert aux secteurs public, privé et associatif et qui encourage des échanges entre ces secteurs ;

- une promotion plus rapide à l'intérieur du Civil Service et la fin de la titularisation (tenure) des hauts fonctionnaires ;

- un Civil Service capable d'être leader, avec des compétences de leadership dans la gestion et la réalisation des projets ;

- une approche plus stratégique et innovante ;

- une organisation de l'État autour des problèmes à résoudre en non pas une organisation des problèmes autour de l'État.

Compte tenu de ces principes, les tendances à la dispersion géographique et à la marchandisation de l'administration centrale se poursuivront dans les années à venir. En particulier, suite à un audit sur l'efficacité dans le Civil Service (le Rapport Gershon de 2004), le chancelier de l'Échiquier, Gordon Brown, a annoncé, dans son discours du Budget de mars 2004, la suppression de 30000 postes au ministère du Travail et des Retraites (Department of Work and Pensions) avant 2008, ainsi que la suppression de 45000 postes dans les deux directions de la collecte des impôts directs et indirects ${ }^{17}$, qui seront fusionnées. Il a déclaré que le gouvernement souhaite mobiliser les nouvelles technologies de l'information et de la communication pour diminuer les effectifs dans les services de traitement (back office) de l'administration centrale, afin d'augmenter les effectifs directement en relation avec le public et de ramener les coûts d'administration 
de $5 \%$ à $3,7 \%$ du budget public, d'ici $2008^{18}$. De même, le gouvernement compte diminuer les coûts de fonctionnement grâce à la délocalisation des services vers les régions.

\section{Le nouveau modèle du Civil Service}

La restructuration du Civil Service depuis 1979 s'inscrit dans une politique globale lancée par la Nouvelle droite pour restreindre l'action des services publics et la rendre plus économe, plus efficiente et plus efficace - les « $3 \mathrm{E} »$ (economy, efficiency and effectiveness). Les nouvelles pratiques de fonctionnement, qui sont développées à l'intérieur du Civil Service, font également partie d'une "Nouvelle gestion publique» (New Public Management), par laquelle les comportements des organismes publics sont de plus en plus calqués sur ceux du secteur privé.

Toutefois, les réformes et la nouvelle gestion publique suscitent de nombreuses interrogations sur la capacité des organismes et des employés publics à agir dans l'intérêt collectif. De même, ils soulèvent certaines questions constitutionnelles quant au contrôle de l'appareil de l'État par les politiques et à la nouvelle relation qui s'est forgée entre le citoyen et les pouvoirs publics.

\section{La Nouvelle gestion publique}

24 L'éclatement de la structure du Civil Service et la contractualisation des relations en son sein et avec le public font partie de la restructuration de tout le secteur public britannique, depuis 1979. Dans certains domaines, cette restructuration a été réalisée par la privatisation des services publics (télécommunications, gaz, etc.). Pour les services non marchands (les services qui ne sont pas vendus auprès des utilisateurs/ clients) comme l'éducation et la santé, les gouvernements conservateurs ont introduit des réformes similaires. Celles-ci donnent une plus grande autonomie opérationnelle aux prestataires de services (les écoles, les universités, les hôpitaux, etc.), qui doivent dorénavant agir à l'instar des entreprises privées. Ils continuent à recevoir leur financement des autorités publiques, mais les fonds dont dispose chaque prestataire sont liés à sa capacité de satisfaire la demande des usagers ou "consommateurs " (parents d'élèves, patients, etc.). Les prestataires de service sont incités à entrer en concurrence pour attirer des « clients » et donc attirer des fonds publics. Ils acquièrent une grande autonomie budgétaire, ce qui leur permet de - et les oblige à - gérer leur personnel, leurs investissements, leurs actifs immobiliers etc.

Le leitmotiv de ces restructurations est l'application aux services publics des modes de gestion caractérisant l'entreprise privée. Par l'élaboration d'objectifs précis liés à des budgets bien définis, les gouvernements de Thatcher et Major ont cherché à rendre l'utilisation des fonds publics plus claire et à dépolitiser l'action publique par le biais de la recherche d'un meilleur rapport qualité-prix (value for money) des prestations.

En développant des mécanismes de concurrence à l'intérieur de secteur public, ils ont cherché à mobiliser la logique de la maximisation (des bénéfices) de l'entreprise privée afin de rendre les organismes publics plus dynamiques et entreprenants et donc plus efficients et efficaces dans l'utilisation des ressources. Pris dans leur ensemble, les 
réformes des services publics ont donc donné naissance à une "Nouvelle gestion publique $»^{19}$.

\section{Les nouvelles structures de gouvernance}

27 Toutefois, l'éclatement de la structure du Civil Service (et celle des autres services publics) provoque des problèmes de coordination, comme nous l'avons vu précédemment. Une grande partie des efforts entrepris par les gouvernements Blair pour améliorer l'action publique cherche à résoudre ces problèmes. Comment assurer le fonctionnement des services de sorte que la volonté politique des élus soit respectée et que les services publics correspondent effectivement aux attentes et aux droits des citoyens?

Historiquement, la subordination de l'administration au pouvoir public et l'organisation des services publics, en Grande-Bretagne et dans les autres pays industrialisés, sont fondées sur des structures hiérarchiques et bureaucratiques ainsi que sur l'application des règles administratives: c'est le cas du Civil Service dont la structure uniforme, hiérarchique et bureaucratique est développée délibérément durant la première moitié du $\mathrm{XX}^{\mathrm{e}}$ siècle. Les réformes Thatcher et Major, par contre, se sont surtout appuyées sur les préceptes de la concurrence et des lois du marché. Or, dans de nombreux cas, le modèle du marché - qui suppose une transaction monétaire, la présence d'un " grand nombre » de vendeurs et d'acheteurs, des produits homogènes et des informations sur les conditions du marché qui sont gratuites et largement disponibles - s'applique mal aux services publics ${ }^{20}$. Le marché n'assure donc pas toujours une production et une consommation de services efficients ou efficaces. De même, les lois du marché n'assurent pas généralement une distribution de ressources qui soit équitable et qui prenne en compte une certaine égalité de droits juridiques, politiques et sociaux définissant la notion de citoyenneté. Enfin, la cessation d'activité pour raison de faillite, l'ultime sanction du marché, n'existe pas pour les organismes publics. Les autorités publiques sont alors obligées d'intervenir dans le fonctionnement et dans l'utilisation des services pour pallier les carences du marché.

La restructuration du Civil Service par la création des agences d'exécution a donc fini par mener au développement d'autres instruments de coordination qui dépassent l'opposition conventionnelle entre hiérarchies et marchés ${ }^{21}$. En particulier, il est devenu habituel de constater que la « nouvelle gestion publique » est aujourd'hui alliée à de nouvelles structures de "gouvernance». Celles-ci permettent aux autorités politiques de définir les contours et le contenu de l'action publique et de déterminer les ressources qui sont allouées aux services, sans nécessairement s'occuper de leur réalisation. De même, les autorités publiques doivent vérifier que le travail réalisé par des prestataires publics, privés ou associatifs de services correspond effectivement à leurs missions. Il est clair que la contractualisation des relations entre les autorités politiques et les prestataires de services (entre les ministères de tutelle et les agences d'exécution dans le cas du Civil Service) joue un rôle déterminant dans ces nouvelles structures de gouvernance. Le processus qui va de l'élaboration des objectifs de service à la sanction en cas de non-respect des objectifs - en passant par l'adéquation des ressources attribuées aux prestataires avec les services à réaliser et la vérification des services effectivement fournis - est donc devenu fondamental à l'exercice du pouvoir politique. 
30 Selon R.A.W. Rhodes, cette nouvelle gouvernance se fonde alors sur la coordination par des réseaux de l'élaboration de la politique publique (policy networks) ${ }^{22}$. De tels réseaux qui lient acteurs politiques, prestataires de services (publics ou privés), groupes de pression, etc. existent depuis longtemps en Grande-Bretagne. La « Nouvelle droite » a même critiqué leur façon de défendre des intérêts particuliers et cherché à réduire leur impact sur la politique publique (c'était surtout le cas des syndicats). Mais la fragmentation des services publics a créé de nouveaux réseaux et renforcé certains réseaux existants. Gérer ces réseaux (l'enjeu de la gouvernance) demande d'autres techniques et d'autres valeurs comparées à la coordination des activités par des hiérarchies ou par le marché. Pour R.A.W. Rhodes et alii, la caractéristique-clé des réseaux est le rôle joué par la confiance (trust) réciproque des acteurs, une confiance fondée sur des valeurs et des normes partagées ${ }^{23}$. Ce n'est que par la confiance réciproque que les acteurs peuvent réaliser des services publics efficacement, leurs relations étant fondées sur des engagements communs et gérées par la "diplomatie » et non pas par des relations marchandes.

Tableau 2 : Les caractéristiques clés des marchés, des hiérarchies et des réseaux

\begin{tabular}{|l|l|l|l|}
\hline & Marchés des & Hiérarchies & Réseaux \\
\hline $\begin{array}{l}\text { Fondement contrat et les droits de } \\
\text { relations }\end{array}$ & $\begin{array}{l}\text { une relation d'emploi } \\
\text { direct }\end{array}$ & $\begin{array}{l}\text { échange des } \\
\text { ressources }\end{array}$ \\
\hline Degré de dépendance & indépendant & dépendant & inter-dépendant \\
\hline $\begin{array}{l}\text { Support de l'échange } \\
\text { Modes de résolution } \\
\text { de conflits et de } \\
\text { coordination }\end{array}$ & $\begin{array}{l}\text { négociation du prix et } \\
\text { poursuites judiciaires }\end{array}$ & règles et ordres & diplomatie \\
\hline Culture & concurrence & subordinationce \\
\hline
\end{tabular}

Source : R.A.W. Rhodes, P. Carmichael, J.McMillan \& A. Massey, op.cit., p.27.

31 C'est précisément le maintien et le renouvellement de tels réseaux qui est l'une des priorités principales de la politique travailliste en matière de réforme des services publics. Dans le cas du Civil Service, l'audit des agences a bien montré les problèmes de coordination entre les ministères et les agences d'exécution et a justement préconisé des mesures pour combler ces lacunes. Il s'agit en effet de trouver des moyens institutions, procédures et pratiques communes - qui répondent aux défaillances de la coordination basée sur la contractualisation, moyens qui renforcent la coopération entre les différentes parties de l'administration. Pourtant, la poursuite des réformes annoncées en 2004, dans le but de réaliser d'importantes économies, augmentera ces problèmes de coordination.

32 Le gouvernement travailliste semble se trouver face à une situation paradoxale. D'un côté, la contractualisation et la marchandisation des services publics (y compris le Civil service) engendrent des problèmes de coordination, dans la mesure où la contractualisation sépare les intérêts spécifiques des uns et des autres. Pour y 
remédier, le gouvernement Blair souhaite développer des mécanismes de coordination en réseau. De l'autre côté, il entend renforcer l'ouverture du Civil Service aux acteurs externes, allant jusqu'à supprimer la titularisation (tenure) des hauts fonctionnaires. La poursuite de cette politique d'ouverture risque d'augmenter la contractualisation et la marchandisation des relations à l'intérieur du Civil Service. Les orientations du gouvernement Blair continuent donc à être contradictoires.

\section{La portée constitutionnelle de la création des agences d'exécution}

La création des agences d'exécution, dans le cadre des autres réformes du Civil Service mises en place depuis 1979, a aussi eu plusieurs conséquences constitutionnelles. Elle a changé la distribution du pouvoir à l'intérieur du gouvernement, renforçant le rôle du pouvoir exécutif central (core executive). Elle a aussi modifié le fonctionnement constitutionnel du Civil Service lui-même. Plus généralement, la contractualisation des relations entre les citoyens et les services publics fait partie d'une modification de la citoyenneté en Grande-Bretagne.

Il est habituel aujourd'hui de constater que les réformes de l'État et des services publics entreprises depuis la fin des années 1970 ont renforcé le contrôle du « pouvoir exécutif central " (core executive) sur les services publics au moment même où les véritables prestataires de services - hôpitaux, écoles et agences d'exécution, etc. - sont devenus autonomes. Il s'agit ici surtout des divers réseaux et institutions qui mènent et coordonnent les politiques principales du gouvernement, au centre desquels se trouvent les services du Premier ministre (son bureau privé, son bureau politique, son service de presse et la cellule politique ${ }^{24}$ ) et le Cabinet Office (qui gère le fonctionnement du Cabinet, les comités du Cabinet et qui coordonne les activités du Civil Service en général). Leur importance dans l'élaboration de la politique publique a augmenté, surtout aux dépens des institutions intermédiaires de l'État (notamment le Civil Service et les collectivités locales).

L'autre institution clé du pouvoir exécutif central est le Trésor, dont les instruments pour maîtriser les dépenses publiques ont été renforcés tout au long des années 1980 et 1990. Plus précisément, on constate que la dispersion de la responsabilité opérationnelle dans le secteur public - y compris les agences d'exécution - a été accompagnée par de nouveaux mécanismes de contrôle budgétaire. Ces mécanismes ont consolidé le pouvoir financier du gouvernement central. Les gouvernements Blair ont eux aussi renforcé ces capacités de gestion du Trésor grâce, par exemple, aux PSA, qui stipulent très précisément les objectifs principaux des services publics et les ressources disponibles pour les réaliser. Dans le cas précis des agences d'exécution, les « secrétaires d'État au Trésor » (Treasury Ministers) doivent approuver leurs documents cadres. Ils doivent de même approuver les objectifs établis pour les agences et enfin, ils doivent donner leur accord pour les plans d'entreprise et les plans d'affaires (corporate and business plans) des agences (Cabinet Office).

Par ailleurs, l'importance attachée dorénavant à la gestion des agences a diminué, en partie, le rôle joué par le Civil Service dans l'élaboration de la politique publique. Son élaboration par l'administration centrale est aussi limitée par le fait que les gouvernements de Margaret Thatcher ont eu recours à des conseillers spéciaux (special advisers) pour formuler leurs politiques. De plus, l'intégration de tels conseillers externes au Civil Service dans l'élaboration de la politique publique s'est fortement 
développée sous les gouvernements Blair, ce qui appelle une nouvelle réglementation de leurs activités : une proposition de loi sur le Civil Service est actuellement en cours d'adoption par la Chambre des Lords. Nous n'entrerons pas ici dans le débat sur la place constitutionnelle de tels conseillers, mais nous constatons simplement que les politiques se tournent de plus en plus vers des conseillers externes au Civil Service pour élaborer leurs programmes. De fait, ce rôle historique de l'administration centrale a tendance à diminuer.

La création des agences d'exécution a également suscité, dans un premier temps, quelques craintes concernant la responsabilité des ministres devant le Parlement (accountability to Parliament) pour le fonctionnement de leurs services. Il s'agit d'un principe fondamental de la démocratie représentative britannique, qui a été dilué au moment de la création des agences et qui était bafoué par certains ministres pendant les années 1990. Pour remédier à ces problèmes, les directeurs d'agence peuvent être appelés à témoigner devant les Commissions parlementaires. Ils doivent également donner des réponses écrites aux questions parlementaires, réponses qui sont publiées dans le Hansard.

Plus généralement, il est à noter que la mise en œuvre de la politique publique et les activités du Civil Service sont devenues plus transparentes. Nous ne détaillerons pas ce phénomène ici, mais nous constatons que les informations sur l'action gouvernementale accessibles aux politiques et au public se sont largement développées depuis les années 1980. Auparavant, l'administration centrale britannique était très marquée par le respect du secret de l'État, incarné par la loi sur les secrets d'État de $1913^{25}$. Mais la création des agences et la contractualisation des relations entre organismes publics, l'élaboration des indicateurs de performance, l'utilisation des nouvelles technologies de l'information et de la communication ont tous contribué à rendre le fonctionnement du Civil Service plus transparent. Ces changements ont aussi été accompagnés par la révision de la loi sur les secrets d'État en 1989, puis l'adoption en 2000 de la loi sur la liberté d'information ${ }^{26}$, qui doit entrer en vigueur en 2005.

Cependant, les restructurations des services publics depuis 1979 sont souvent critiquées pour avoir affaibli la notion de citoyenneté. Non seulement certains services publics, et surtout des prestations sociales, ont été réduits ou supprimés, contribuant ainsi à la montée des inégalités, mais les réformes des années 1980 et 1990 ont restreint la citoyenneté à une conception plutôt marchande et individualiste. Le discours sur l'efficacité et la nécessité de faire face à la demande des « consommateurs » des services publics ont eu tendance à appauvrir les dimensions politiques et sociales de la citoyenneté. Cette logique est tout à fait cohérente avec l'extension des lois du marché pour coordonner les activités des agents privés et publics. Mais le fait de définir la citoyenneté sur des bases contractuelles, voire marchandes, vide la citoyenneté et le domaine public d'une partie de son contenu ${ }^{27}$. Si le secteur public ne devient qu'un simple prestataire de biens et de services (éducation, santé, logement, etc.), quel est l'intérêt pour le citoyen de s'adresser aux instances publiques pour satisfaire ses besoins particuliers?

\section{Conclusion}

40 La création des agences d'exécution, dans le cadre d'un programme plus large de réforme, a profondément changé le fonctionnement du Civil Service. Ce programme 
lancé par les gouvernements Thatcher a été repris et perfectionné par ses successeurs. Les actions des gouvernements Blair ont largement entériné les réformes conservatrices dans ce domaine. Certes, les travaillistes ont modifié quelques aspects de la réforme du secteur public et leur discours envers les employés des services publics est beaucoup plus conciliant que celui des conservateurs. Mais pour le Civil Service, leurs projets d'avenir annoncent la poursuite des réformes entamées depuis 1979.

Par conséquent, le Civil Service s'éloigne de plus en plus de son modèle historique. La nature hiérarchique et uniforme de l'administration centrale a cédé la place à une organisation hétéroclite, ouverte et interdépendante avec le secteur privé. Les réformes entreprises ont permis une amélioration de la productivité du Civil Service. Son fonctionnement est, dans une certaine mesure, plus en phase avec les attentes des citoyens-consommateurs et il est devenu plus transparent. Mais l'indépendance et la cohésion du Civil Service et donc son rôle constitutionnel ont été réduits et sa place dans la politique publique britannique diminuée.

\section{BIBLIOGRAPHIE}

BLAIR, T. 'Speech by the Prime Minister The Right Honourable Tony Blair MP at the Civil Service Reform, Delivery and Values event, 24 February 2004'. <http://www.civilservice.gov.uk/reform/ documents/PM_speech.rtf>, 4 nov. 2004.

CABINET OFFICE. Governance Arrangements in the UK Part I : Executive (or 'Next Steps') Agencies. <http://www.cabinet-office.gov.uk/agencies-publicbodies/ execagencies.htm>, 8 avril 2004.

CABINET OFFICE. Guidance on Framework Documents. December 2002, <http://www.cabinetoffice.gov.uk/agencies-publicbodies/guiddepts/docs/NewFrameworkDocGuidance-version2.pdf>, 28 avril 2004.

EFFICIENCY UNIT. Improving Management in Government : The Next Steps (Ibbs Report). London: HMSO, 1988.

FARNHAM, D., \& HORTON, S., (eds). Managing the New Public Services. Basingstoke: Macmillan, 1993.

HM TREASURY. (1998b), Public Services for the Future : Modernisation, Reform, Accountability, (Comprehensive Spending Review : New Public Spending Plans 1999-2002). December 1998, Cmnd 4181, London: The Stationery Office.

HM TREASURY. Competing for Quality. London, HMSO, Cmnd 1730, 1991.

HUGHES, O. Public Management and Administration : An Introduction. $2^{\text {nd }}$ edition, Basingstoke, Macmillan, 1998.

LE GRAND, J. ; BARTLETT, W. et al. Quasi-Markets and Social Policy. Basingstoke: Macmillan, 1993.

OPSR - The Prime Minister's Office of Public Service Reform. Better Government Services : Executive Agencies in the 21 st century, "The Alexander Report”, à la suite du Agency Policy Review, juillet 2002. <http://www.cabinet-office.gov.uk/agencies-publicbodies/>, 10 avril 2004. 
PRIME MINISTER and MINISTER FOR THE CABINET OFFICE. Modernising Government. Cmnd 4310, London: The Stationery Office, March 1999.

PRIME MINISTER. Efficiency and Effectiveness in the Civil Service. Cmnd 8186, London, HMSO, 1982.

PRIME MINISTER. The Civil Service : Continuity and Change. London: HMSO, Cmnd 2627, 1994.

RHODES, R.A.W. Understanding Governance : Policy Networks, Governance, Reflexivity and Accountability. Buckingham: Open University Press, 1997.

RHODES, R.A.W. ; CARMICHAEL, P. ; McMILLAN, J. \& MASSEY, A. Decentralizing the Civil Service : From unitary State to differential Polity in the United Kingdom. Buckingham: Open University Press, 2003.

WILLIAMSON, O. Markets and Hierarchies. New York: Free Press, 1975.

WRIGHT, T. Citizens and Subjects : An Essay on British Politics. London: Routledge, 1993.

\section{NOTES}

1. M. THATCHER, The Downing Street Years, London: Harper Collins, 1993, p. 48.

2. Il s'agit d'audits internes de l'administration menés par Derek Raynor et son équipe. Celui-ci est directeur de Marks \& Spencer, en détachement auprès des services du Premier ministre où il dirige la célèbre Cellule d'efficience (Efficiency Unit). Les responsables de services de l'administration sont invités à solliciter ses conseils sur la manière d'améliorer le fonctionnement et la performance de leurs services.

3. HOUSE OF LORDS, HL 55, Public Service-Report, Report from the Public Service Committee, House of Lords, session 1997-1998, London: TSO, 1998, § 63.

4. EFFICIENCY UNIT, Improving Management in Government : The Next Steps, (Ibbs Report), London: HMSO, 1988, p. 1.

5. Ibid, p. 5.

6. OPSR - The Prime Minister's Office of Public Service Reform: Better Government Services: Executive agencies in the $21^{\text {st }}$ century, "The Alexander Report", à la suite de l'Agency Policy Review, juillet 2002, <http://www.cabinet-office.gov.uk/agencies-publicbodies/>, 10 avril 2004,p. 9.

7. S. LEWIS \& J. JONES, 'The Use of Output and Performance Measurement in Government Departments', pp 39-55, in CAVE, M. ; KOGAN, M. \& SMITH, R., (eds), Output and Performance Measurement in Government : The State of the Art, London: Kingsley, 1990, pp. 39-55.

8. The Citizen's Charter : Raising the Standard, Cmnd 1599, London: HMSO, 1991.

9. CABINET OFFICE, The Next Steps Report 1997, Cmnd 3889, London: TSO, 12 March 1998, chap. 3.

10. HM TREASURY, Competing for Quality, London: HMSO, Cmnd 1730, 1991, p. 1.

11. Ibid, p. 12.

12. J. TONGE, The New Civil Service, Tisbury: Baseline Books, 1999, pp. 67-68.

13. HM TREASURY, Modern Public Services for Britain: Investing in Reform, (Comprehensive Spending Review : New Public Spending Plans 1999-2002), July 1998, Cmnd 4011, London: TSO.

14. PRIME MINISTER and MINISTER FOR THE CABINET OFFICE, Modernising Government, Cmnd 4310, London: TSO, March 1999, pp. 1-2.

15. Ibid, part $3, \S 8$.

16. Op.cit. OPSR, pp. 7-8.

17. Il s'agit du Inland Revenue pour la collecte des impôts directs et de Customs and Excise pour la collecte des impôts indirects.

18. HM TREASURY, (2004), Chancellor of the Exchequer's Budget Statement, 17 March 2004, <http:// www.hm-treasury.gov.uk/>, 30 avril 2004. 
19. D. FARNHAM \& S. HORTON, (eds), Managing the New Public Services, Basingstoke: Macmillan, 1993, pp. 34-54 ; W. WALDEGRAVE, Commons Hansard, Debates, colonne 1028, 26/11/1992, <http:// www.parliament.the-stationery-office.co.uk/pa/cm199293/cmhansrd/1992-11-26/

Debate-4.html>, 12 oct 2004 ; O. HUGHES, Public Management and Administration : An Introduction, $2^{\text {nd }}$ edition, Basingstoke: Macmillan, 1998.

20. J. LE GRAND ; W. BARTLETT et al, Quasi-Markets and Social Policy, Basingstoke: Macmillan, 1993, pp. 13-34.

21. Cette opposition est d'abord théorisée par O. WILLIAMSON, Markets and Hierarchies, New York: Free Press, 1975.

22. R.A.W. RHODES, Understanding Governance: Policy Networks, Governance, Reflexivity and Accountability, Buckingham: Open University Press, 1997, p. 37.

23. RHODES, R.A.W. ; CARMICHAEL, P.; MCMILLAN, J. \& MASSEY, A., Decentralizing the Civil Service: From unitary State to differential Polity in the United Kingdom, Buckingham: Open University Press, 2003, pp. 26-28.

24. Private Office, Political Office, Press Office, Policy Unit.

25. Official Secrets Act, 1913.

26. Freedom of Information Act, 2000.

27. T. WRIGHT, Citizens and Subjects: An essay on British Politics, London: Routledge, 1993, pp. 121-127.

\section{RÉSUMÉS}

Cet article détaille les réformes du Civil Service effectuées depuis 1979 et notamment la place centrale qu'occupe la création des agences d'exécution. Il analyse comment ces agences et l'adoption de nouvelles pratiques de gestion et de gouvernance ont contribué à la mise en place d'un nouveau modèle de l'administration centrale britannique, caractérisé par une certaine décentralisation et marchandisation des services. L'article analyse aussi les conséquences constitutionnelles de ces réformes.

This article reviews the reforms of the Civil Service which have taken place since 1979 and the central role played by the creation of executive agencies. It analyses how the development of executive agencies along with the adoption of new management and governance practices have contributed to the emergence of a new model of Britain's central government administration, characterised by a certain decentralisation and commodification of services. The article also analyses the constitutional consequences of these reforms.

\section{AUTEUR}

\section{NICHOLAS SOWELS}

Université Paris I Panthéon-Sorbonne 